\title{
010 EARLY CHILDHOOD IS OVERRATED-A LIFE COURSE PERSPECTIVE USING SIBLINGS AND POPULATIONS
}

E Wall-Wieler, LL Roos, D Chateau. Manitoba Centre for Health Policy, Department of Community Health Sciences, Faculty of Medicine, University of Manitoba

\subsection{6/jech-2014-205217.10}

Purpose/Rationale for the project How important are early childhood predictors in the presence of later childhood and early adolescent predictors when examining several late adolescent health and education outcomes? How well do the models work? A sibling design examines a set of time-varying predictors on two health outcomes (ADHD/Conduct Disorders, asthma) between ages 14 and 18 and one education outcome (failure to graduate high school), controlling for a variety measures.

Methods Multi-level modeling of a sample of randomly selected consecutive siblings and twins $(n=29,444)$ born in Manitoba, Canada between 1984 and 1989 allowed comparing family and individual level characteristics. Extensive sensitivity testing involved comparison with a population sample $(n=62,820)$. This analysis uses files from the Population Health Research Data Repository at the Manitoba Centre for Health Policy (MCHP), linked across ministries, which include information on individual level health and education. Census data on neighborhood household income, education and so forth, were also incorporated.

Results/Next steps Although several 0-3 variables were significant for the education outcome, very few were significant for the health outcomes indicating that early childhood variables are not particularly important in predicting late adolescent health and education outcomes. Time-varying predictors in early childhood were markedly less important when including later childhood (48) and early adolescent (9-13) health predictor; however, events in early childhood might be important in embedding specific outcomes in later life. Using a life course approach provided an excellent fit for the externalizing mental conditions model $(c$-statistic $=0.826)$ and a reasonable fit for failure to graduate high school $(c$-statistic $=0.793)$ and asthma $(c$-statistic $=0.798)$ models. This paper adds to the growing literature that suggests a stronger focus be put on adolescence as a "second sensitive developmental period" when examining late adolescent and adult outcomes. Future research should be less concerned with birth and early childhood predictors and put a stronger focus on the late childhood and early adolescent time periods, as they bring with them a period of rapid brain maturation that can significantly modify childhood trajectories. 